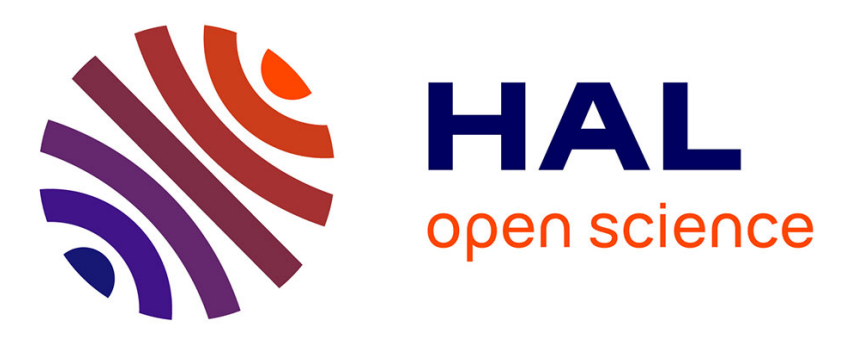

\title{
Guarded Attribute Grammars and Publish/Subscribe for implementing distributed collaborative business processes with high data availability
}

\author{
Maurice Tchoupé Tchendji, Joskel Ngoufo Tagueu
}

\section{To cite this version:}

Maurice Tchoupé Tchendji, Joskel Ngoufo Tagueu. Guarded Attribute Grammars and Publish/Subscribe for implementing distributed collaborative business processes with high data availability. 2020. hal-02924307

\author{
HAL Id: hal-02924307 \\ https://hal.science/hal-02924307 \\ Preprint submitted on 27 Aug 2020
}

HAL is a multi-disciplinary open access archive for the deposit and dissemination of scientific research documents, whether they are published or not. The documents may come from teaching and research institutions in France or abroad, or from public or private research centers.
L'archive ouverte pluridisciplinaire HAL, est destinée au dépôt et à la diffusion de documents scientifiques de niveau recherche, publiés ou non, émanant des établissements d'enseignement et de recherche français ou étrangers, des laboratoires publics ou privés. 


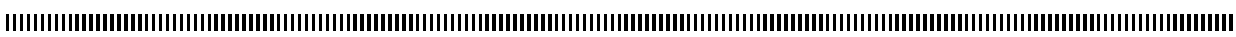

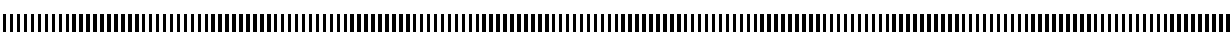
|

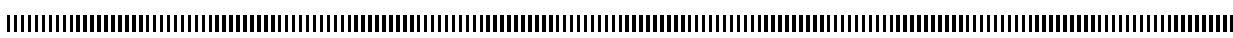

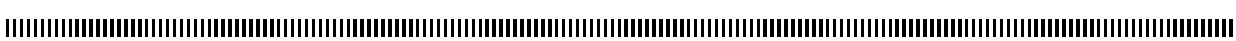
| | |

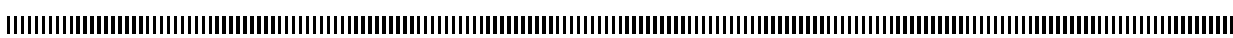

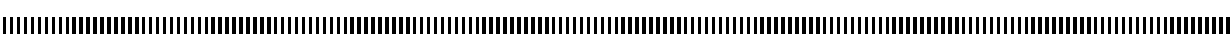

\title{
Guarded Attribute Grammars and Publish/Subscribe for implementing distributed collaborative business processes with high data availability
}

\author{
Maurice TCHOupé TCHENDJI ${ }^{1}$ and Joskel NgOufo TAgueU ${ }^{2}$ \\ Department of Mathematics and Computer Science \\ University of Dschang, PO Box 67, Dschang-Cameroon \\ LIRIMA, FUCHSIA associate team \\ 1 maurice.tchoupe@univ-dschang.org, ttchoupe@yahoo.fr \\ 2 jngoufotagueu@yahoo.com
}

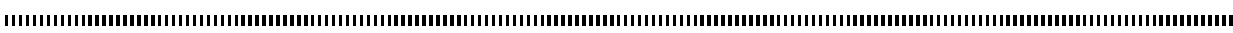

ABSTRACT. With the ever-increasing development of the Internet and the diversification of communication media, business processes of companies are increasingly collaborative and distributed. This contrasts with traditional solutions deployed for their management which are usually centralized, based on the activity flow or on the exchanged documents. Moreover, the users who are usually the main actors in collaborations are often relegated to second place. Recently, a distributed, data-driven and user-centric approach called Guarded Attributed Grammar (GAG) has been proposed for the modeling of such processes; it thus provides an answer to most of these limitations. In this paper, we present an approach for implementing business processes modeled using GAG in which communications are done by publish/subscribe with redirection of subscription (pub/sub-RS). The pub/sub-RS - which we propose - guarantees high data availability during the process execution by ensuring that an actor, perceived as a subscriber, will always receive a data he needs to perform a task as soon as it is produced. Moreover, if the data is semi-stuctured, and produced collaboratively and incrementally by several actors, its subscribers will be notified as soon as one of its components (a prefix) is produced simultaneously, as they will be subscribed in a transparent way to the remaining components (the suffix).

RÉSUMÉ. Avec le développement toujours croissant de l'Internet et la diversification des moyens de communication, les processus métiers sont de plus en plus collaboratifs et distribués. Ceci contraste avec les solutions traditionnelles déployées pour leur gestion qui sont habituellement centralisées, basées sur le flux d'activités ou sur les documents échangés. Bien plus, les utilisateurs qui sont généralement les acteurs principaux dans la collaboration y sont souvent relégués au second rang. Récemment, une approche distribuée, centrée sur l'utilisateur et pilotée par les données appelée Grammaires Attribuées Gardées (GAG), a été proposée pour la modélisation de tels processus; elle fournit donc une réponse à la plupart de ces limitations. Dans ce papier, nous présentons une approche de mise en œuvre de processus métiers modélisés à l'aide des GAG dans laquelle les communications se font par publish/subscribe avec redirection de souscriptions (pub/sub-RS). Le pub/sub-RS (que nous proposons) garantit une haute disponibilité des données pendant l'exécution d'un processus en assurant qu'un acteur (perçu comme un abonné) recevra toujours une donnée dont il a besoin pour effectuer une tâche dès qu'elle est produite. De plus, si la donnée est semi-structurée et produite collaborativement et incrémentalement par plusieurs acteurs, ses abonnés seront notifiés dès qu'une de ses composantes (un préfixe) est produite en même temps qu'ils seront abonnés de manière transparente aux composantes résiduelles (le suffixe).

KEYWORDS : Collaborative business processes, GAG, Artifact, Publish/Subscribe, Subscription redirection, Semi-structured data, Service oriented computing, Software architecture

MOTS-CLÉS : Processus métiers collaboratifs, GAG, Artefact, Publish/Subscribe, Redirection de souscriptions, Données semi-structurées, Calcul orienté service, Architecture logicielle

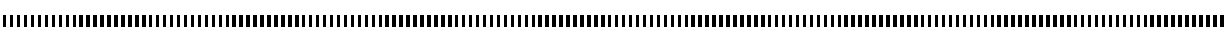




\section{Introduction}

Business processes are processes that represent the activities of companies. Their purpose is to orchestrate activities that contribute to the achievement of organizational goals. The ever-increasing development of the Internet and the diversification of means of communication has led to the emergence of new needs, including the need for distributed process execution. Most business tasks in large organizations are performed collaboratively by actors possibly positioned in remote geographical locations, requiring therefore, the need for rapid information transfer for consistent decision-making.

Generally, collaborative business process management models are either based on the process activity flow $[12,11]$; or on the documents exchanged during the process $[13,6$, 14]; or on both, as in models centered on the artifact ${ }^{1}[8,4,10,5]$. A disadvantage of these models is that they model the users of the process as second-class actors, when they are not simply ignored. Indeed, although they are very often the main actors of collaboration, they are usually modelled as plain resources performing specific tasks in a context [3]. It is entirely appropriate that collaborative business process models explicitly highlight the roles played by users, as long as their implications would be predominant in collaboration: this is what Badouel et al. propose in the GAG (Guarded Attribute Grammars) model $[3,2]$, which is a grammatical approach to model collaborative, distributed, data-driven and user-centric business processes.

Intuitively, a GAG is a collection of semantic rules ${ }^{2}$ or business rules describing for a business process how to produce data (synthesized attribute values) from information of the environment (inherited attribute values and user inputs). In the GAG execution model, the artifacts are used to represent and manage the flow of activities, the data and the life cycle of processes. They are intentionally modeled by trees whose nodes represent the tasks and have attributes to contain all the information about a process from its creation in the system to its completion. These nodes can be divided into two subsets: the one of the closed nodes corresponding to the completed tasks, and the one of the open nodes corresponding to the pending or running tasks. The choice of how to perform a task associated with a given open node is left to the discretion of the user, who performs it by selecting one of the business rules (from the GAG) applicable to that open node (see section 2). The business rules applicable to an open node at any given time are a function of data previously generated in the process, and data provided by users: it is in this sense that the GAG model is said to be data-driven and user-centric.

The objective of this paper is to present an approach for implementing distributed collaborative business processes modeled using $\mathrm{GAG}^{3}$ and communicating through a new variant of publish/subscribe ${ }^{4}$ called publish/subscribe with redirection of subscriptions abbreviated as $p u b / s u b-R S$. We are therefore specifically interested in collaborative business processes involving several actors located on different geographical sites who work in parallel and communicate asynchronously. With the pub/sub-RS protocol, we want to

1. "An artifact is a document that conveys all the information concerning a particular case from its inception in the system until its completion. It contains all the relevant information about the entity together with a lifecycle that models its possible evolutions through the business process" [3]. So we can assimilate it to an active document [1] combining data and processing.

2. A variant of the semantic rules of attribute grammars.

3. In the rest of this manuscript, we will name "GAG processes" business processes modeled using GAG.

4. The publish/subscribe is an asynchronous communication scheme between publishers and subscribers. Subscribers express their interest in data through subscriptions and publishers publish data for subscribers. A subscriber is notified each time a publication corresponds to a subscription he has made. 
guarantee a high availability of information (even if it is only partially produced) in order to allow the actors to start processing as soon as possible.

Paper contributions: the majors contributions of this paper are the proposal of a new variant of publish/subscribe communication protocol called publish/subscribe with redirection of subscriptions (pub/sub-RS), the proposal of a scheme of implementation of GAG processes communicating by pub/sub-RS, and an approach for deploying such an implementation on a component-based architecture. The pub/sub-RS allows the exchange of potentially semi-structured data (whose components can eventually be produced by different peers) asynchronously, incrementally and without intermediaries ${ }^{5}$.

Manuscript organization: section 2 provides an overview of business process modeling with GAG; some formal definitions are also given. The pub/sub-RS protocol as well as our approach to implementing the coupled model GAG - Pub/sub-RS is presented in section 3. Section 4 presents how to deploy and execute GAG with pub/sub-RS on a distributed component-based architecture. Section 5 is devoted to the conclusion.

\section{Business process modeling with GAG}

In this section, we present some fundamental concepts of the GAG model for business process modeling. The interested reader can find a more complete presentation of GAG in $[3,2]$.

\subsection{Business process, business rule and artifact}

A business process can be interpreted as a task $\left(t_{0}\right)$ to be executed which, depending on its complexity, can be branched/decomposed into subtasks $\left(t_{1}, \ldots, t_{n}\right)$ potentially executed by different actors. Conceptually, this decomposition can be modeled by a rule called business rule that can be represented by a production of the form $P: s_{0} \rightarrow s_{1} \ldots s_{n}$ expressing the fact that the service $s_{0}$ to be invoked to execute the task $t_{0}$ must invoke the (sub)services $s_{1}, \ldots, s_{n}$ which are the services to be invoked to execute the subtasks $t_{1}, \ldots, t_{n}$ of $t_{0}$ respectively. We call s-production a production having $s$ on the left hand side. For a same service $s$, we can have several applicable business rules (i.e. several ways to perform a task) and therefore several s-productions. In this case, the choice of the business rule to be applied is up to the actor of the process responsible for executing the task associated with the service (reminder: the execution of a GAG is user-centric).

Each process is associated with an artifact modeled by a tree whose nodes are sorted. We write $X:: s$ to indicate that the artifact node $X$ is of sort $s$; this means that it is an instance of the service $s$. An artifact is defined by a set of equations of the form $X=$ $P\left(X_{1}, \ldots, X_{n}\right)$, indicating that $X:: s$ is a node labeled by the production $P: s \rightarrow s_{1} \ldots s_{n}$ and has as successors, the nodes $X_{1}:: s_{1}, \ldots, X_{n}:: s_{n}$. A node $X:: s$ that is not defined by any equation is called open node ${ }^{6}$; it corresponds to a pending task and will have to be refined (i.e. extended into a subtree, see figure 1) by applying a production corresponding to its sort $s$ (a s-production $P: s \rightarrow s_{1} \ldots s_{n}$ ).

This refinement allows to "close" the node $X$ which is now defined by the equation $X=P\left(X_{1}, \ldots, X_{n}\right)$ such that $X_{1}, \ldots, X_{n}$ are new open nodes created; they have for respective sorts $s_{1}, \ldots, s_{n}$ (see figure 1 ).

5. A data produced by a peer $X$ to a peer $Y$ is sent directly to it without passing through a peer $Z$.

6 . In the tree representation of the artifact it is a leaf not labeled by a production. 
To execute a process, we start from an initial artifact reduced to an open node (of the sort of one of the axioms, see definition 2) and refine it by successive application of business rules, until we obtain an artifact containing only closed nodes: it is said closed (closed artifact).

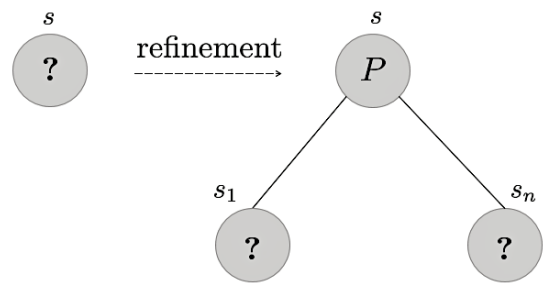

Figure 1. Refinement of an open node [2]

\subsection{Data flow and configuration of a GAG process}

To model the data exchanged between the different services associated with open nodes, more information is attached to the open nodes using attributes. Each sort $s$ is then equipped with a set of inherited attributes and a set of synthesized attributes whose values are terms defined over a ranked alphabet. Recall that a term, in this case, is either a variable or an expression of the form $c\left(t_{1}, \ldots, t_{n}\right)$ where $c$ is a $n$ rank symbol, and $t_{1}, \ldots, t_{n}$ are terms. The inherited attributes represent the input values of the services and the synthesized attributes represent the output values. Taking into account the attributes, the complete form of specifying a business rule is as follows:

$$
\begin{aligned}
s_{0}\left(p_{1}, \ldots, p_{n}\right)\left\langle u_{1}, \ldots, u_{m}\right\rangle \rightarrow & s_{1}\left(t_{1}^{(1)}, \ldots, t_{n_{1}}^{(1)}\right)\left\langle y_{1}^{(1)}, \ldots, y_{m_{1}}^{(1)}\right\rangle \\
& \ldots \\
& s_{k}\left(t_{1}^{(k)}, \ldots, t_{n_{k}}^{(k)}\right)\left\langle y_{1}^{(k)}, \ldots, y_{m_{k}}^{(k)}\right\rangle
\end{aligned}
$$

where $p_{j}, u_{j}$, and $t_{j}^{l}$ are terms and $y_{j}^{l}$ are variables. This new form allows, in addition to representing the ramification of a service $s_{0}$ into services $s_{1}, \ldots, s_{n}$, to also specify the existing dependencies between their data. For example, the rule $s_{0}\left(\boldsymbol{p}_{\mathbf{0}}(x, y)\right)\left\langle\boldsymbol{u}_{\mathbf{0}}(z, t)\right\rangle \rightarrow$ $s_{1}()\langle z\rangle s_{2}(x)\langle t\rangle$ means that the service $s_{0}$ must invoke the services $s_{1}$ and $s_{2}$ by providing $s_{2}$ with the parameter $x$; much more, these services must respectively return the $z$ and $t$ values after their execution. Let's now introduce the notion of form which offers a simpler notation for writing business rules.

Definition 1. A form of sort $s$ is an expression $F=s\left(t_{1}, \ldots, t_{n}\right)\left\langle u_{1}, \ldots, u_{m}\right\rangle$ where $t_{i}$ and $u_{j}$ are terms. The terms $t_{1}, \ldots, t_{n}$ (resp. $u_{1}, \ldots, u_{m}$ ) give the values of the inherited (resp. synthesized) attributes attached to the form $F$. A form of sort $s$ is noted $F:: s$.

With this notion, the rule of the equation 1 for example can be rewritten simpler as follows: $F_{0} \rightarrow F_{1} \ldots F_{n}$ where the $F_{i}$ are forms. Moreover, it also allows us to define more formally a GAG and its configuration at a given time as follows:

Definition 2. (Guarded Attribute Grammar (GAG)). Given a set of attribute sorts $S, a$ GAG $G$ is defined by a set $\mathcal{R}$ of rules $R: F_{0} \rightarrow F_{1} \ldots F_{k}$ such that $F_{i}:: s_{i}\left(s_{i} \in S\right)$ are forms, and a set of sorts $A \subseteq S$ called axioms: $G=(\mathcal{R}, \mathcal{A})$. A sort is used (resp. defined) in $G$ if it appears in the right (resp. left) hand side of a rule. Axioms are the sorts that are 
defined, but not used; they correspond to the services for starting processes. The sort that are used, but not defined, are called terminals; they correspond to external services. We note $N$ the set of defined sorts and $T$ the set of terminals.

Definition 3. (Configuration of a GAG). A Configuration $\Gamma$ of a $G A G G=(\mathcal{R}, \mathcal{A})$ is a set of sorted nodes $X$ where each node is associated with an equation written in one of the following two forms depending on whether the node is closed or open: for closed nodes, $X=R\left(X_{1}, \ldots, X_{k}\right)$ with $R \in \mathcal{R}$ and $X_{1}, \ldots, X_{n}$, the successor nodes of $X$. For open nodes, $X=s\left(t_{1}, \ldots, t_{n}\right)\left\langle x_{1}, \ldots, x_{k}\right\rangle$ where $X$ is of sort $s ; t_{1}, \ldots, t_{n}$ are terms representing the values of the inherited attributes of $X$, and $x_{1}, \ldots, x_{k}$ are variables associated with the synthesized attributes of $X$.

Roughly speaking, the configuration is an artifact in which data are explicitly represented. With the concept of configuration, the execution of a GAG process consists in starting from an initial configuration reduced to an open node (of the type of one of the axioms), and making it evolve (move from one configuration to another) by applying a business rule to one of its open nodes. The operation is reiterated until a configuration with only closed nodes is obtained.

\subsection{Example of GAG process: the process of submitting a thesis defense application in a doctoral school}

In this sub-section, we present an example of the use of GAG to model the process of submitting a thesis defense application in a doctoral school. This example is strongly inspired by what is done in the doctoral school of the Faculty of Science of the University of Dschang (Cameroon). We will get back to this example later to illustrate our implementation scheme with pub/sub-RS in a distributed component-based architecture (section 4).

Example 1. When a student of the Dschang University Doctoral School (called Dschang School) has finished writing his thesis, he prepares a defense application file and submits it to his home department. There, the department carries out a set of processing operations at the end of which it is either rejected or passed on to the hierarchy. In order to be validated, the file must successively be approved by the services of the Department, the Dschang School, the DAAC ${ }^{7}$ and the Rectorate. At the end of the tour, the student is notified of the Rectorate's decision and can, in case of rejection, introduce a request at the Department level. One may wish to carry out this process in such a way as to inform the student as soon as a new treatment is applied to his file.

The example 1 can be modeled using GAG by considering that: 1) it is a distributed process involving 5 actors; 2) all the processing carried out by the actors on the file is considered as composite information, the components of which are produced incrementally by each actor at the appropriate time (on receipt of the file) and disseminated to the other actors, according to their needs. The tables 1 to 5 present the GAGs specifying the behaviors of the different actors in the process. The global GAG defining the process is composed ${ }^{8}$ of these different GAGs. In the modeling of these behaviors, we consider that

7. Division des Affaires Académiques et de la Coopération (Academic and Cooperation Affairs Department).

8. The GAG composition operator noted $\oplus$ is defined in [3]. It allows to create a GAG $G$ called Global GAG from a set of local GAGs $G_{1}, \ldots, G_{k}: G=G_{1} \oplus \ldots \oplus G_{k}$. 
the outputs of treatments are stored incrementally in a term of the algebraic type ${ }^{9}$ list $^{10}$. The objective is to have, at any time, on each site, a list containing incrementally, all the outputs of treatments that have already been applied and which are of it (the site) interest: each service (Department, Dschang School, DAAC or Rectorate) concatenating the result of its treatment.

Remark 1. In the specifications of the tables 1 to 5 , we use parametric rules. The values of the parameters will be provided by the actor applying the rule. For example, to apply the rule RectorateDecision of the table 5, the rector must provide the value of the parameter decision.

Table 1. Local GAG of student InitApplication [application] $\quad: \quad$ Submission ()\langle\rangle$\rightarrow$

DepartmentService(application) $\langle$ processing $\rangle$

InitRequest [applicationId, attachments] $\quad: \quad \operatorname{Request}()\langle\rangle \rightarrow$

DepartmentRequest (applicationId, attachments) $\langle$ processing $\rangle$

Table 2. Local GAG of department

DptProcessing $[$ processing $] \quad$ : $\quad$ DepartementService $($ application $)\langle\operatorname{Cons}($ processing,$n)\rangle \rightarrow$ DschangSchoolService ( processing, application) $\langle n\rangle$

Request : DepartmentRequest(applicationId, attachments) $\langle$ processing $\rangle \rightarrow$ DepartmentService $($ new (applicationId, attachments)) $\langle$ processing $\rangle$

DptRejection[rejectionReasons] $\quad: \quad$ DepartmentService $($ application $)\langle\operatorname{Cons}($ rejectionReasons, $\mathbf{N i l})\rangle \rightarrow \varepsilon$

Table 3. Local GAG of Dschang School

DschProcessing [ processing] : $\quad$ DschangSchoolService $($ D ptP, application $)\langle$ Cons $($ processing,$n)\rangle \rightarrow$ DaacService ( processing, application) $\langle n\rangle$

DschRejection[rejectionReasons] $\quad: \quad$ DschangSchoolService $($ DptP, application $)\langle$ Cons $($ re jectionReasons, Nil $)\rangle \rightarrow \varepsilon$

Table 4. Local GAG of DAAC

DaacProcessing [processing] :

DaacRejection[rejectionReasons] :
DaacService $($ Dsch, application $)\langle$ Cons $($ procesing,$n)\rangle \rightarrow$

RectorateService $($ processing, application $)\langle n\rangle$

DaacService $($ DschP, application $)\langle$ Cons $($ re jectionReasons, $\mathbf{N i l})\rangle \rightarrow \varepsilon$

Table 5. Local GAG of Rectorate

RectorateDecision $[$ decision $] \quad: \quad$ RectorateService $($ DaacP, application $)\langle\operatorname{Cons}($ decision, $\mathbf{N i l})\rangle \rightarrow \varepsilon$

9. An algebraic type is a composite data type, which combines the functionalities of product types (tuplets or records) and sum types (disjoint union). In combination with recursivity, it is used to express structured data such as lists and trees.

10. Reminder: The list type is defined by two constructors: Nil, which allows to create an empty list, and Cons which allows to create a new list by adding an element to a pre-existing list. 


\section{Implementation of distributed GAG processes communicating by pub/sub-RS}

\subsection{The publish/subscribe with redirection of subscriptions: an overview}

In a distributed context, each actor participates in the process from his site, applying locally and asynchronously the business rules of his local GAG. Note that for a given actor, his local GAG defines the services of the process that he provides in the form of axioms, the way he proceeds to execute these services in the form of business rules, and the services of other actors that he may need in the form of terminals. As the work is distributed and asynchronous, an actor may be responsible for executing a service for which certain input data must be provided by other actors (they correspond to some outputs of their services). Similarly, he may also be responsible for the execution of services whose output data are expected by other actors. To ensure the efficient and without intermediaries exchange of (semi-structured) data between actors, we have designed an asynchronous protocol for data exchange between actor based on the publish/subscribe called publish/subscribe with redirection of subscriptions in short pub/sub-RS.

The pub/sub-RS is a new variant of the publish/subscribe protocol particularly suitable for the exchange of semi-structured data whose components can be produced incrementally by different actors. As in the classic publish/subscribe, any actor has the possibility to subscribe to one or more events in order to be notified as soon as a publisher generates an occurrence of an event corresponding to one of his subscriptions [7]. However, unlike the traditional publish/subscribe, in the pub/sub-RS, since the exchanged data are potentially semi-structured and can be produced incrementally (by collaboration of several actors), they are also delivered incrementally. In fact, as soon as a prefix $x$ of a data to which an actor has subscribed is produced, it is immediately sent to him simultaneously as he (the actor) is subscribed (subscription redirection) in a transparent way to the residue (the suffix) of the initial data.

The pub/sub-RS's operating mode is therefore as follows: each time an actor needs a data to be produced by another actor, he must subscribe to it; if it is produced incrementally, the prefix produced must each time be sent to him at the same time as he is subscribed to the residue. More concretely, if an actor $A$ needs a data $d_{b}$ to be produced by an actor $B$, then a subscription of $A$ to the data $d_{b}$ must be stored on the actor $B$ 's site in a subscription list provided for this purpose and, $A$ will be notified as soon as $B$ produces $d_{b}$. If $d_{b}$ is semi-structured and is produced incrementally, then $A$ will be notified as soon as $d_{b}$ components are produced. For example, if $d_{b}$ is a list of the form $d_{b}=d_{1} b: d_{s} b$, then $A$ will be notified as soon as $d_{1} b$ is generated by $B$ and automatically subscribed to the residual data $d_{s} b$ (which will not necessary be produced by $B$ ). It is the fact that an actor $A$ subscribes to a data $d$ and is then automatically and transparently subscribed to the residual data of $d$ ( $d_{s} b$ in the current example) that we call redirection of subscriptions.

\subsection{Workspace of an actor}

An actor's workspace is represented by two data structures: the local configuration $\Gamma$ of his local GAG and the list of subscriptions on the data he must produce called $L S$ (figure 2 shows an illustration of a workspace). The local configuration of an actor $\Gamma$ informs him about the tasks in which he participates and the data necessary to perform them. Thus, all the data he handles are associated with local variables of his configuration. However, in case of sharing data, a same data can be associated with many variables 
belonging to different configurations. We say in that case that those variables have the same publication identifier. We define the publication identifier of a variable $x$-noted $\dot{x}$ - as the global unique name of the data intended to be stored there. For example, in the previous description (section 3.1), the data $d_{b}$ can be stored in the configuration of $A$ in the variable $x_{a}$ and in the configuration of $B$ in the variable $x_{b}$; the variables $x_{a}$ and $x_{b}$ in that case must have the same publication identifier $\left(\dot{x_{a}}=\dot{x_{b}}\right)$ since they correspond to local names of $d_{b}$.

The subscription list $L S$ is the set of subscriptions on the data that the actor must produce and publish. A subscription is a pair of the form $(\dot{x}, b)$ where $\dot{x}$ is the global unique name (i.e a publication identifier) of the data to which the subscription relates and $b$ is the identifier of the remote actor who subscribes.
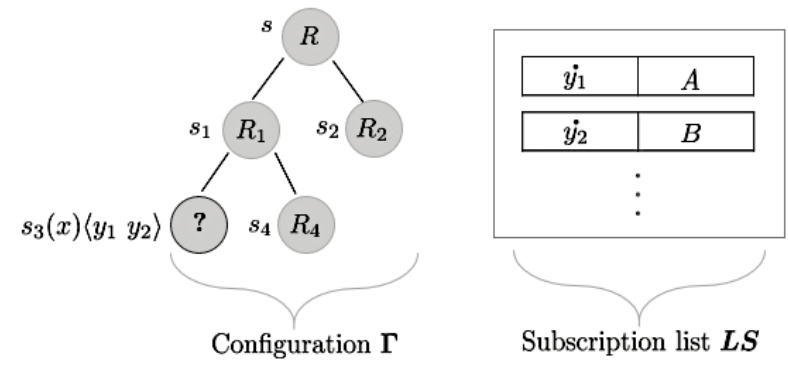

Figure 2. Illustration of an actor's workspace with two subscriptions on output value

\subsection{Local application of a business rule}

Each actor in the process works locally using the business rules contained in his local GAG. In the following, we will assimilate an actor to the site on which he operates by designating him by the identifier of his site. Let's consider a site named $a$ with a configuration $\Gamma$ and a list of subscriptions $L S$. Let's also consider $X=F$ an open node of $\Gamma$ and $R=F_{0} \rightarrow F_{1} \ldots F_{k}$ a business rule such that $F_{i}^{\prime} s$ variables are disjoint from those of $\Gamma$. Apply a business rule $R$ to $X$ is equivalent to sequentially perform the following four operations (see figure 3): 1) update the local configuration $\Gamma ; 2$ ) notify the sites subscribing to the data that have been produced; 3 ) update the subscription list $L S$; 4) call remote services (if necessary). Let's now precisely present the treatments carried out by each of these operations:

(1) Update from $\Gamma$ to $\Gamma^{\prime}$. The application of $R$ to the node $X$ refines it into open successor nodes $X_{1}, \ldots, X_{n}$ associated with sub-services $s_{1}, \ldots, s_{n}$ of $F_{1}, \ldots, F_{n}$. Thus, it is necessary to add to the configuration the new nodes created and close the node $X$. The new configuration is then $\Gamma^{\prime}$ :

$$
\begin{aligned}
\Gamma^{\prime}= & \left\{X=R\left(X_{1}, \ldots, X_{k}\right)\right\} \\
\cup & \left\{X_{1}=F_{1} \sigma, \ldots, X_{k}=F_{k} \sigma\right\} \\
\cup & \left\{X^{\prime}=F \mid\left(X^{\prime}=F\right) \in \Gamma, X^{\prime} \neq X\right\}
\end{aligned}
$$


where $\sigma=$ match $^{11}\left(F_{0}, X\right)$ is a substitution that matches the input values of $F_{0}$ to the input values of $F$ and the output values of $F$ to the output values of $F_{0}$. For each variable $x$ of the forms $F_{i}, 0 \leq i \leq n$, we assign its name to the data that will be stored there: $\dot{x}=x$. To ensure that this name is globally unique, one can simply use a local name generator that creates variable names prefixed by the site identifier.

Remark 2. We consider that a business rule $R$ is only applicable to an open node $X=$ $s\left(t_{1}, \ldots, t_{n}\right)\left\langle y_{1}, \ldots, y_{m}\right\rangle$ if the $t_{i}, 1 \leq i \leq n$ are completely defined values, i.e. no longer contain variables.

(2) Subscriber notifications. The application of the rule allows new data to be generated using substitution $\sigma$. An equation of the form $(x=t)$ in $\sigma$ means that the variable $x$ is now assigned the value $t$. By doing so, all sites that have subscribed to the data represented by $x$ must be notified. These are the sites $b$ as it exists a subscription $(\dot{x}, b)$ in $L S$. All notifications are therefore: $N S=\{(x=t, b) \mid(x=t) \in \sigma$ and $(\dot{x}, b) \in L S\}$, where each element $(x=t, b)$ is a notification meaning that the site $b$ (when $b \neq a$ ) will receive the equation $(\dot{x}=\operatorname{global}(t)) ; x$ is the (local) variable associated with the data to which $b$ had subscribed and $t$ is its value; the function global $(t)$ renames the variables in $t$ by their publication identifiers: $\operatorname{global}(t)=t[y / \dot{y}]$. When for an element $(x=t, b)$ of $N S$ we have $b=a$, no message is sent, it means that the current site $a$ has produced a data to which it is itself subscribed. In that case, we simply update the local configuration with the value of $x: \Gamma^{\prime}=\Gamma[y / t \mid \dot{y}=\dot{x}]$.

(3) Update of the subscription list $L S$ to $L S^{\prime}$. Once the notifications have been made, the local subscription list must be updated: subscriptions on data that have already been sent to subscribers $(O L S)$ are removed, and new subscriptions $(N L S)$ from sites related to dependencies between the data defined in the rule are added. The new local subscription list on the site $a$ is therefore: $L S^{\prime}=L S \backslash O L S \cup N L S$ where

$$
\begin{aligned}
O L S & =\{(\dot{x}, b) \mid(x=t, b) \in N S\} \text { and } \\
N L S & =\bigcup_{X_{i}: s_{i}, s_{i} \in N} N L S\left(X_{i}\right) .
\end{aligned}
$$

The $X_{i}$ in $N L S$ are new created nodes that have to be refined by the current site. $N L S\left(X_{i}\right)$ is the set of subscriptions on the data to be produced by a new non-terminal node $X_{i}$. It is defined by: $N L S\left(X_{i}\right)=\bigcup_{x \in \text { out }\left(X_{i}\right)}\left\{(\dot{x}, b) \mid b \in S U B S_{R, \sigma, N S}(x)\right\}$ where out $(X)$ is the set of variables associated with the synthesized attributes of $X$ and $S U B S_{R, \sigma, N S}(x)$ is the set of sites that need the value of a variable $x$; the following paragraph describes how this set is constructed.

Computation of subscriber sites to a variable. The set of sites to be subscribed to a variable $\mathrm{x}$ of a new node is computed from three parameters: the business rule $R$ whose application allowed to create the variable $x$, the substitution $\sigma$ produced following the application of the rule and all notifications $N S$ generated by the application of $R$. It is noted $S U B S_{R, \sigma, N S}(x)$ and is created from the combination of three sets:

11. The function match is defined in [3]. It returns a substitution $\sigma$ itself consisting in two main substitutions $\sigma_{\text {in }}$ and $\sigma_{\text {out }}$. For a specific refinement of a node $X=F$ into successor nodes $X_{1}=F_{1}$ to $X_{n}=F_{n}$ via a rule $F_{0} \rightarrow F_{1} \ldots F_{n}, \sigma_{\text {in }}$ matches the input values of $F_{0}$ to the input values of $F$ and $\sigma_{\text {out }}$ matches the output values of $F$ to the output values of $F_{0} . \sigma=\sigma_{\text {out }} \cup \sigma_{\text {in }} \sigma_{\text {out }}$ [3]. 


$$
\begin{array}{rll}
\operatorname{SUBS}_{R, \sigma, N S}(x) & =\{\text { owner }(x)\} \\
& \cup \quad \operatorname{BROTHER}_{R, \sigma}(x) \\
& \cup \operatorname{REDIRECT} T_{N S}(x)
\end{array}
$$

with :

- owner $(x)$ : the site that created the variable $x$. We consider that each site is interested in the variables it creates. For a variable $\mathrm{x}$ created on a site a, we define the function owner $(x)=a$. Variables are created during business rule applications, service call and notification receptions.

- BROTHER $_{R, \sigma}(x)$ : all the sites that will execute a service with $\mathrm{x}$ as input variable. If $R=F_{0} \rightarrow F_{1} \ldots F_{k}$ then all the new nodes created by the rule application are $N O D E_{R, \sigma}=$ $\left\{X_{i}=F_{i} \sigma \mid 1 \leq i \leq k\right\}$ and we have $\operatorname{BROTHER}_{R, \sigma}(x)=\left\{b \mid(X=F) \in N O D E_{R, \sigma}, x \in\right.$ $\operatorname{in}(X)$, provider $(X)=b\}$ where $\operatorname{in}(X)$ is the set of inherited attributes (input variables) of $X$. For a node $X:: s$, provider $(X)$ returns the identifier of the site that provides the service $s$ (it is equal to the current site when $s$ is a defined sort).

$-R E D I R E C T_{N S}(x)$ : the set of subscriptions created by the redirection of subscriptions. Since the redirection of subscriptions occurs when a data $d$ to which sites had subscribed is partially produced, sites that had subscribed to $d$ (they are in $N S^{12}$ ) must therefore be subscribed to its residual data. If the term $t$ is the partial produced value of $d$, then the residual data of $d$ correspond to the variables of $t$ : $\operatorname{REDIRECT}_{N S}(x)=\{b \mid(y=t, b) \in N S, x$ is a variable of $t\}$.

(4) Remote service calls. For each new open node created $X:: s$ such that $s$ is a terminal, a service call must be made to the site providing the service $s$ ( $s$ is an axiom of its local GAG). This service call must also contain the subscription list for the data to be produced by the service. A service call therefore consists in sending a message $m=\left(X=F, L S T_{X}\right)$, where $X$ is the node representing the service, $F$ the form associated with $X$ and $L S T_{X}$ the list of subscriptions to be transferred to the site that will refine the node $X$. Service calls to be made are extracted from the set $I=\left\{\left(X_{i}=F_{i} \sigma, L S T_{R, \sigma, N S}\left(X_{i}\right), b\right) \mid X_{i}:: s_{i}\right.$, $s_{i} \in T, b=$ provider $\left.\left(X_{i}\right)\right\}$ where $L S T_{R, \sigma, N S}(X)=\bigcup_{x \in \text { out }(X)}\left\{(\dot{x}, b) \mid b \in S U B S_{R, \sigma, N S}(x)\right\}$.

Each element $(X=F, L S T, b) \in I$ means that the current site $a$ will send the service call $(X=\operatorname{global}(F), L S T)$ to the site $b$.

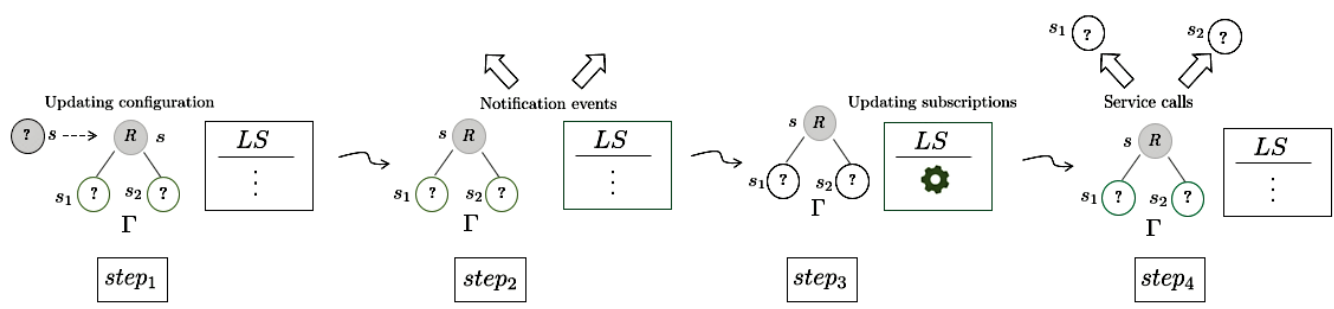

Figure 3. Different execution steps when applying rule

12. Recall that $N S$ is the set of notifications created by the application of the rule. 


\subsection{Processing of messages}

Messages received by a site correspond to service call messages or data production notifications.

When a site receives a service call, $(a)$ it updates its local configuration by creating a new local node matching the remote node received via the service call. The variables created in the new local node have the same publication identifiers as those of their correspondents in the remote node received (this will be used for notifications). After that, (b) subscriptions from remote sites are added to the local subscription list. The following formulas $(f a)$ and $(f b)$ summarize these treatments in equational form:

if $m=\left(Y=s\left(t_{1}, \ldots, t_{n}\right)\left\langle y_{1}, \ldots, y_{q}\right\rangle, L S T_{Y}\right)$ is the service call message received, then :

( fa) $\Gamma^{\prime}=\Gamma \cup\left\{\bar{Y}=s\left(\overline{t_{1}}, \ldots, \bar{t}_{n}\right)\left\langle\overline{y_{1}}, \ldots, \bar{y}_{q}\right\rangle\right\}$ with $\overline{t_{i}}=t_{i}[x / \bar{x}], \bar{Y}$ a new local node matching the node $Y$; the $\bar{x}$ and $\overline{y_{j}}$ are new local variables created such that $\dot{\bar{x}}=x, \dot{\overline{y_{j}}}=y_{j}$;

(fb) $L S^{\prime}=L S \cup L S T_{Y}$.

In the case of receiving a notification, the site merely updates its local configuration using the publication identifier to know which variable of the configuration to update. The following formula summarizes this treatment in equational form:

if $m=(x=t)$ is the notification message received, then:

$-\Gamma^{\prime}=\Gamma[y / \bar{t} \mid \dot{y}=x]$ where $\bar{t}=t[z / \bar{z}]$, the $\bar{z}$ being new variables such that $\overline{\bar{z}}=z$;

$-L S^{\prime}=L S$.

\subsection{Illustration}

In this section we illustrate the use of the pub/sub-RS communication protocol in a GAG process through an example. A visual representation of the effects of the various operations on the sites involved is given in figure 4 .

Example 2. Let's consider a process with five actors $A, B, C, D$ and $E$, such that the actor $A$ has the configuration $\Gamma_{A}=\left\{X=s_{A}()\left\langle d_{A}\right\rangle\right\}$ and the local $G A G G_{A}$ containing a business rule $R=s_{A}()\left\langle\operatorname{sum}\left(d_{B}, 5, d_{C}\right)\right\rangle \rightarrow s_{B}()\left\langle d_{B}\right\rangle s_{C}\left(d_{B}\right)\left\langle d_{C}\right\rangle\left(s_{B}\right.$ provided by $B$ and $s_{C}$ provided by $C$ ). Suppose in addition that the subscription list of $A$ is $L S_{A}=\left\{\left(\dot{d}_{A}, D\right),\left(\dot{d}_{A}, E\right)\right\}$.

If $A$ applies the business rule $R$ on the node $X$ of $\Gamma_{A}$ then we will have $\sigma_{\text {in }}=\emptyset$ and $\sigma=\sigma_{\text {out }}=\left\{d_{A}=\operatorname{sum}\left(d_{B}, 5, d_{C}\right)\right\} ; d_{B}$ and $d_{C}$ being new variables with $\dot{d}_{B}=d_{B}$ and $\dot{d}_{C}=d_{C}$. As $d_{A}$ was partially produced, the actors $D$ and $E$ must be notified of the production of this partial data. To do this, we use the set $N S=\left\{\left(d_{A}=\right.\right.$ $\left.\left.\operatorname{sum}\left(d_{B}, 5, d_{C}\right), D\right),\left(d_{A}=\operatorname{sum}\left(d_{B}, 5, d_{C}\right), E\right)\right\}$ containing the notifications generated by the application of the rule. And then, $D$ and $E$ must be subscribed to the residual data $d_{B}$ and $d_{C}$ of $d_{A}: R E D I R E C T_{N S}\left(d_{B}\right)=R E D I R E C T_{N S}\left(d_{C}\right)=\{D, E\}$.

Since the actor $C$ provides the service $s_{C}$ which has $d_{B}$ in its input, he must be subscribed to $d_{B}:$ BROTHER $R_{R, \sigma}\left(d_{B}\right)=\{C\}$. Finally, the set of subscriptions to the variables $d_{B}$ and $d_{C}$ are $S U B S_{R, \sigma, N S}\left(d_{B}\right)=\{A, C, D, E\}$ and $S U B S_{R, \sigma, N S}\left(d_{C}\right)=\{A, D, E\}$; while the service call to be sent to $B$ and $C$ are respectively $m_{1}=\left(X_{1}=s_{B}()\left\langle\dot{d}_{B}\right\rangle,\left\{\left(\dot{d}_{B}, A\right),\left(\dot{d}_{B}, C\right),\left(\dot{d}_{B}, D\right),\left(\dot{d}_{B}, E\right)\right\}\right)$ and $m_{2}=\left(X_{2}=\right.$ $\left.s_{C}\left(\dot{d}_{B}\right)\left\langle\dot{d}_{C}\right\rangle,\left\{\left(\dot{d}_{C}, A\right),\left(\dot{d}_{C}, D\right),\left(\dot{d}_{C}, E\right)\right\}\right)$. 

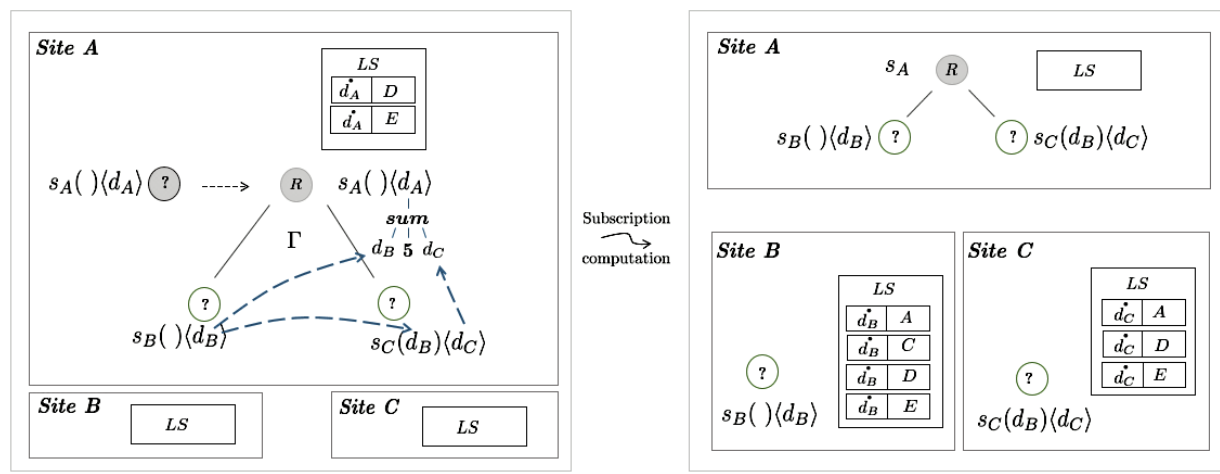

Figure 4. Illustration of the application of a business rule in a distributed context with the pub/sub-RS

\section{Deployment of GAG processes communicating via pub/sub-RS on a distributed component-based architecture}

In this section, we show how to deploy and execute a GAG process using a distributed application made up of a set of peers who communicate across the network. In our proposal each peer hosts an instance of a software component (called in the following $G A G$ software component) from which an actor of the process operates. The actors can thus contribute to the process execution through a dedicated GUI provided by a GAG software component. The underlying idea is to correspond each local GAG of an actor to a software component which allows him to participate to the process. In the following, we present the generic architecture of a GAG software component, as well as its deployment for the execution of an example of a GAG process (the one presented in the example 1).

\subsection{Generic architecture of a peer participant in the execution of a GAG process}

We propose to run a GAG process on a peer-to-peer (P2P) application in which the different peers result from the deployment of a generic component-called GAG software component-on actor's sites. Practically, the architecture of a peer (see figure 5) consists of two main parts: the GAG software component and the peer network interface.

The GAG software component is responsible for the local management of GAG processes in which the local actor is involved. It is made up of four modules (GUI, control, execution and storage). It provides a set of services to other peers and required some services (from other peers) in return. The provided services correspond to the axioms of the local GAG to which is added a notification service ${ }^{13}$ allowing to keep the peer informed as soon as a data to which it had subscribed is produced. The required services correspond to the local GAG terminals.

The GUI module allows an actor to visualize the current states of the processes in which he is involved. It allows him also to contribute to their execution/evolution by applying business rules to his local configuration in accordance with his local GAG.

At the heart of the GAG software component is the execution module whose main role is to manage the life cycle of local GAG process configurations. To do so, it encapsulates

13. The notification service is used by a component $A$ to notify a component $B$, when a business rule applied at the level of the component $A$ produces a data to which the component $B$ had subscribed. 
all the mechanisms for applying business rules, notifications, service calls and redirection of subscriptions. It relies on the storage module to store the data it manages (local GAG, local configurations, subscription list, etc.), and on the control module to send or receive data and commands in formats suitable for their processing.

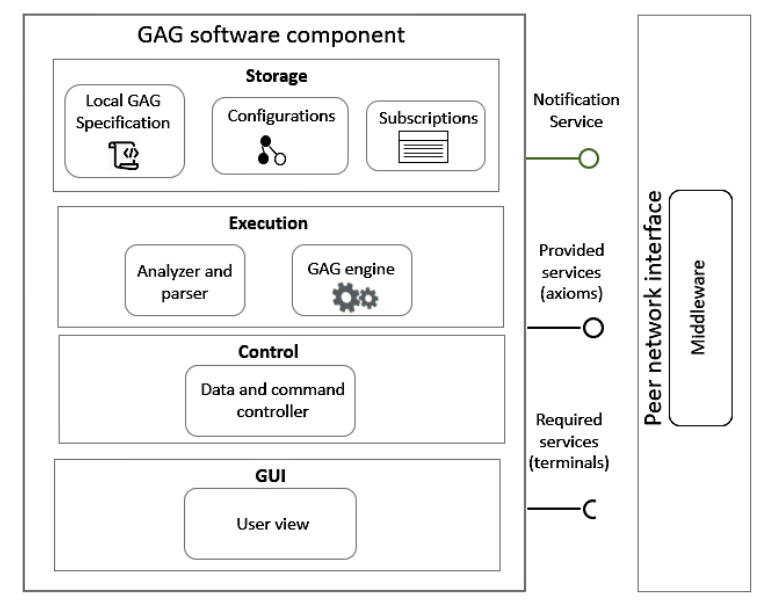

Figure 5. The peer architecture.

Peer-to-peer communication is provided by a middleware ${ }^{14}$ which, by connecting in P2P the GAG software components across the network, allows them to mutually invoke their provided services.

\subsection{Example of deployment of a GAG process: case of the process of processing application files for thesis defense in a doctoral school}

To illustrate the implementation of GAG processes communicating by pub/sub-RS on a component-based architecture, we have developed and deployed the application TinyTSP (Tiny Thesis Submit Process). TinyTSP is a software prototype for managing thesis defense application files in a doctoral school; it is an implementation of the process presented in example 1.

TinyTSP is made of 5 types of peers (see figure 6) corresponding to the 5 types of actors (student, department, Dschang School, DAAC, rectorate) found in the process described in example 1. Each peer hosts an instance of a GAG software component; the local GAG that it encapsulates (see tables 1 to 5) is consistent with the role played by the actor associated with the peer.

TinyTSP provides for each actor a dedicated GUI allowing him to contribute to a process, but also to follow the evolution of an application. The figure 7 for example presents a screenshot of the GUI of submission of an application on a student's site. Once

14. A middleware is third-party software that creates a network for information exchange between different computer applications. For our experiments, we used the SON (Shared-data Overlay Network) middleware: "SON is a generic lightweight P2P middleware that assists application developers by providing an automatic code generation which handles several requirements (e.g., communication mechanisms, message queue management, broadcasting messages, etc.)"[9]. 
an application is submitted, it is sent to the department at the same time as the student subscribes to the outputs of treatments that will be performed on it.

Before producing a data (i.e. applying a treatment to an application file), each actor can find out via a tooltip which actors are subscribed to it. We can observe for example on the figure 8, which presents an execution status of the process on the Dschang School site, that the student and the department are subscribed to the processing that the Dschang School has to perform. Note that the student has been subscribed to it by redirection of subscriptions. The figure 9 shows the status of the process on a student's site after approval by the rectorate.

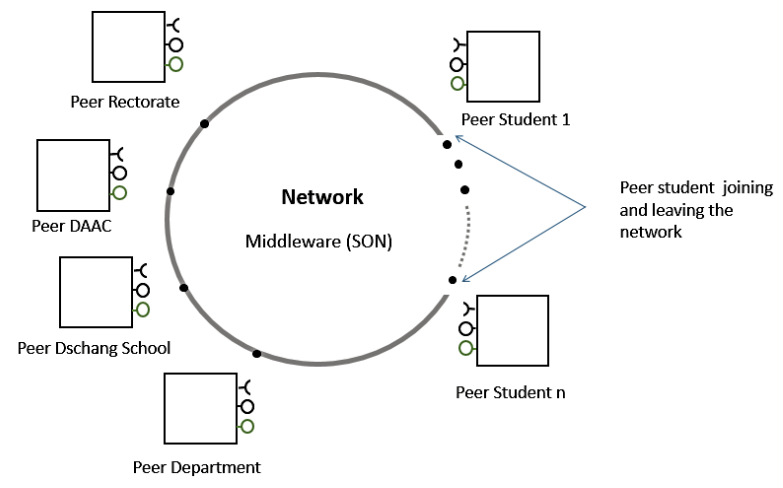

Figure 6. TinyTSP deployment architecture

TinyTSP is implemented in JAVA and the communication between peers is ensured by the SON middleware [9]. The figure 6 presents a synoptic view of its deployment architecture.

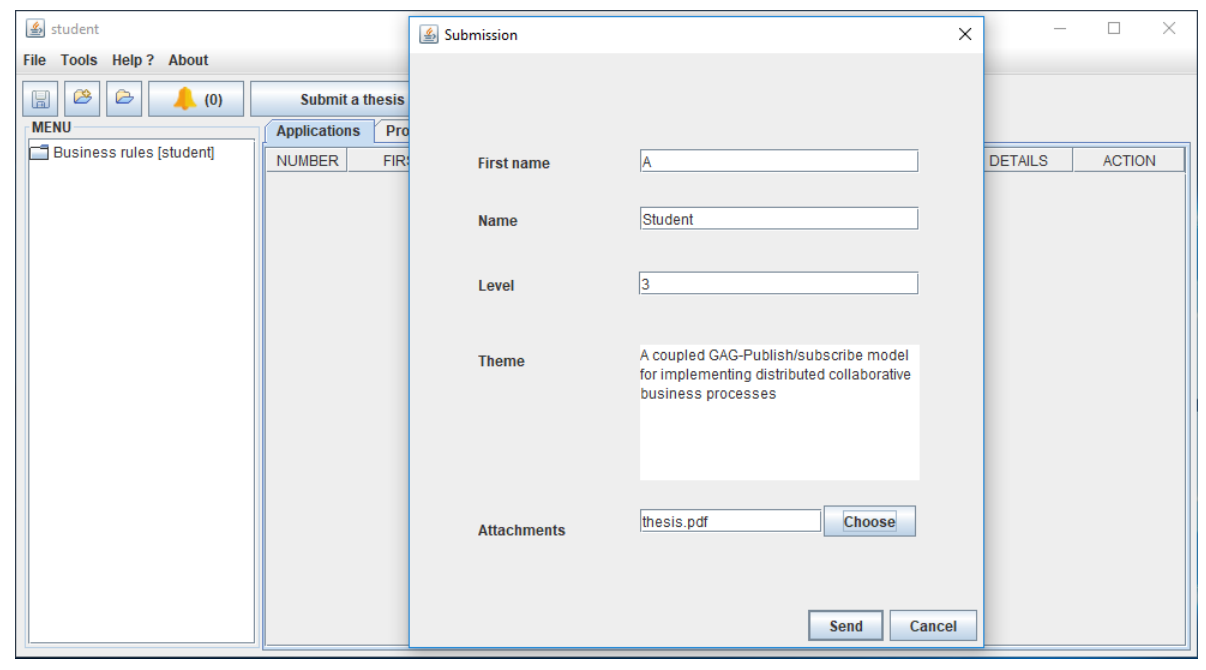

Figure 7. GUI for submitting a file on a student's site 


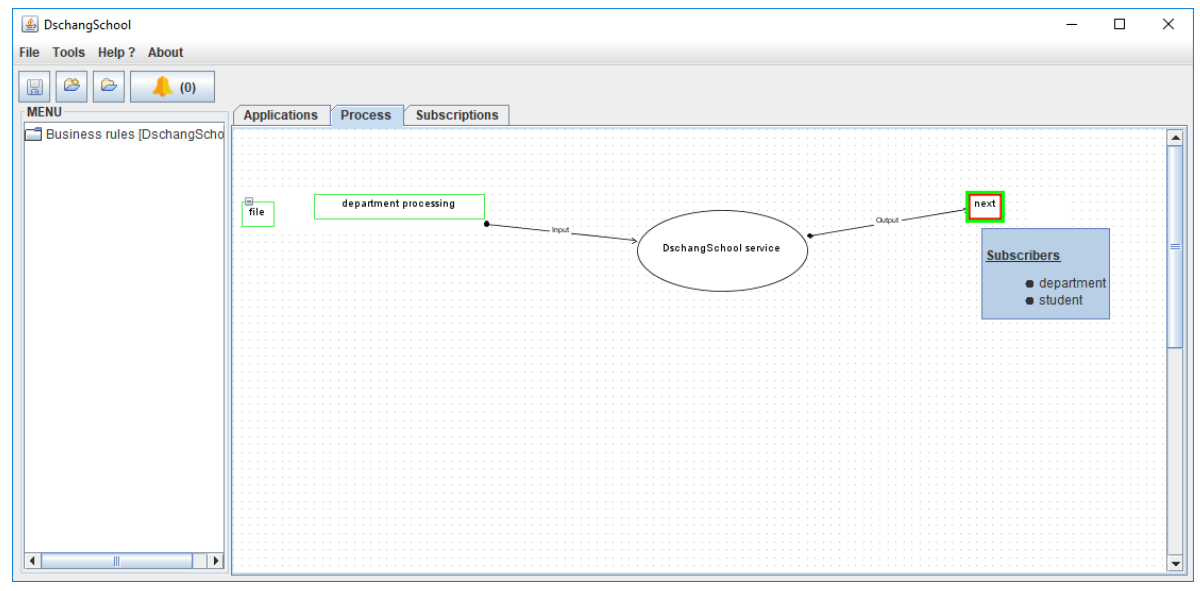

Figure 8. A status of the process when the file arrives at the Dschang School site with a highlight of the subscribers

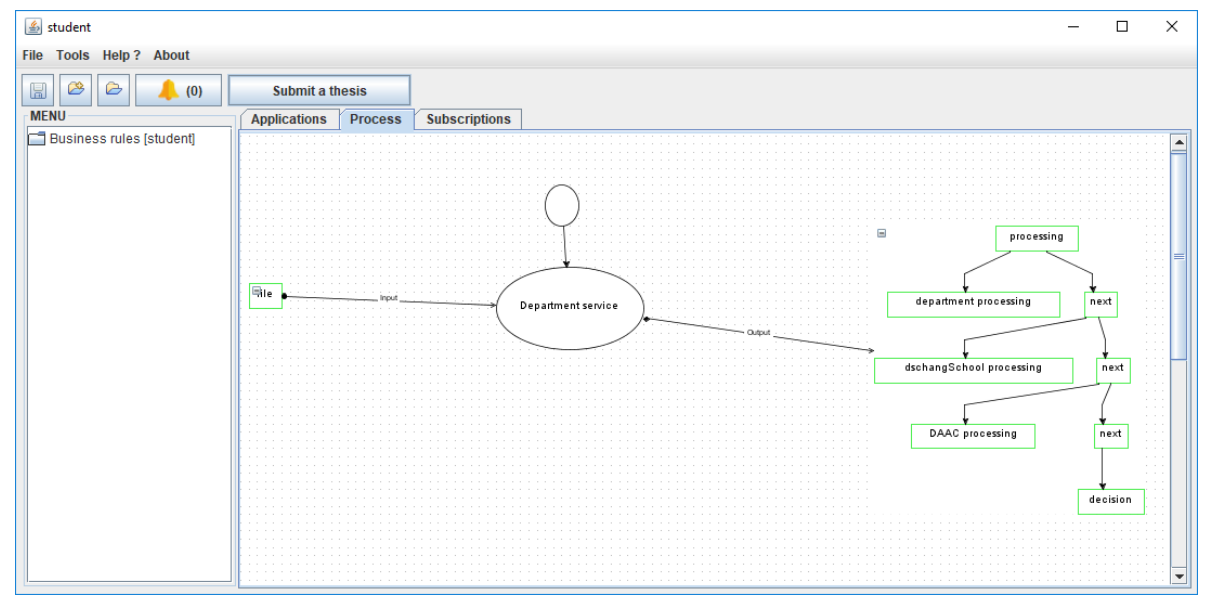

Figure 9. A status of the process on the student site after its approval by the rectorate

\section{Conclusion}

In this paper we have presented an approach of implementing GAG's distributed collaborative business processes in which communication is handled via a new variant of publish/subscribe protocol called publish/subscribe with redirection of subscriptions (pub/sub-RS). The main advantage of this protocol is that, it permits in real time to inform data's subscribers on the evolution of the processing operations by incrementally transferring to them any data they have subscribed to. This can encourage the early initiation of other operations if they are lazy (lazy evaluation): the degree of parallelism and the speed in decision-making are thus improved.

We have also proposed an approach to deploy GAG processes communicating via pub/sub-RS on a component-based architecture. This architecture, as well as the pub/subRS protocol, have been experimented with great satisfaction through the implementation we made of it in a prototype application for managing the process of submitting a thesis 
defense application in a doctoral school. Some screenshots of this application have been presented in this paper. For the development of this application, starting from the textual description of the process, we followed a set of steps (identification of the actors, specification of their local GAGs, development of a GAG engine, etc.) that obviously must be followed identically for the development of any other distributed application using GAG and communicating via pub/sub-RS. The precise presentation of this approach, as well as the production of tools to automate certain steps (for example, the production of the GAG engine) seems to us to be a work worthy of being carried out following this one.

\section{References}

[1] Serge Abiteboul, Omar Benjelloun, and Tova Milo. Positive active xml. In PODS '04: Proceedings of the twenty-third ACM SIGMOD-SIGACT-SIGART symposium on Principles of database systems, pages 35-45, New York, NY, USA, 2004. ACM.

[2] Eric Badouel, Loïc Hélouët, Georges-Edouard Kouamou, and Christophe Morvan. A grammatical approach to data-centric case management in a distributed collaborative environment. In Proceedings of the 30th Annual ACM Symposium on Applied Computing, SAC '15, pages 1834-1839, New York, NY, USA, 2015. ACM.

[3] Eric Badouel, Loïc Hélouët, Georges-Edouard Kouamou, Christophe Morvan, and Nsaibirni Robert Fondze, Jr. Active workspaces: Distributed collaborative systems based on guarded attribute grammars. SIGAPP Appl. Comput. Rev., 15(3):6-34, October 2015.

[4] Elio Damaggio, Alin Deutsch, and Victor Vianu. Artifact systems with data dependencies and arithmetic. ACM Trans. Database Syst., 37(3):22:1-22:36, 2012.

[5] Elio Damaggio, Richard Hull, and Roman Vaculín. On the equivalence of incremental and fixpoint semantics for business artifacts with guard-stage-milestone lifecycles. Inf. Syst., 38(4):561-584, 2013.

[6] J. Dang, C. Toklu, K. Hampel, and U. Enke. Human workflows via documentdriven process choreography. In 2008 International MCETECH Conference on eTechnologies (mcetech 2008), pages 25-33, Jan 2008.

[7] Patrick Th. Eugster, Pascal Felber, Rachid Guerraoui, and Anne-Marie Kermarrec. The many faces of publish/subscribe. ACM Comput. Surv., 35(2):114-131, 2003.

[8] Richard Hull. Artifact-centric business process models: Brief survey of research results and challenges. In On the Move to Meaningful Internet Systems: OTM 2008, OTM 2008 Confederated International Conferences, CoopIS, DOA, GADA, IS, and ODBASE 2008, Monterrey, Mexico, November 9-14, 2008, Proceedings, Part II, pages 1152-1163, 2008.

[9] Ayoub Ait Lahcen and Didier Parigot. A lightweight middleware for developing P2P applications with component and service-based principles. In 15th IEEE International Conference on Computational Science and Engineering, CSE 2012, Paphos, Cyprus, December 5-7, 2012, pages 9-16, 2012.

[10] Anil Nigam and Nathan S. Caswell. Business artifacts: An approach to operational specification. IBM Systems Journal, 42(3):428-445, 2003.

[11] OASIS. Web services business process execution language version 2.0. http: //docs.oasis-open.org/wsbpel/2.0/OS/wsbpel-v2.0-0S.pdf, April 2007. 
[12] OMG. Bpmn specification, business process model and notation. http://www . bpmn. org/.

[13] Nelly Schuster, Christian Zirpins, Stefan Tai, Steve Battle, and Nils Heuer. A service-oriented approach to document-centric situational collaboration processes. In 18th IEEE International Workshops on Enabling Technologies: Infrastructures for Collaborative Enterprises, WETICE 2009, Groningen, The Netherlands, 29 June - 1 July 2009, Proceedings, pages 221-226, 2009.

[14] Lin Zhao, Jianping Xing, and Lingguo Meng. The research and realization of a new workflow model with step-task two layers based on document. In Proceedings of The 1 st IEEE Asia-Pacific Services Computing Conference, APSCC 2006, December 1215, 2006, Guangzhou, China, pages 285-292, 2006. 